This is the final peer-reviewed accepted manuscript of:

Arcidiacono C. et al. (2020) Smart Dairy Farming: Innovative Solutions to Improve Herd Productivity. In: Coppola A., Di Renzo G., Altieri G., D'Antonio P. (eds) Innovative Biosystems Engineering for Sustainable Agriculture, Forestry and Food Production. MID-TERM AllA 2019. Lecture Notes in Civil Engineering, vol 67. Springer, Cham.

The final published version is available online at: http://dx.doi.org/10.1007/978-3030-39299-4 30

Rights / License:

The terms and conditions for the reuse of this version of the manuscript are specified in the publishing policy. For all terms of use and more information see the publisher's website.

This item was downloaded from IRIS Università di Bologna (https://cris.unibo.it/)

When citing, please refer to the published version. 


\title{
Smart Dairy Farming: Innovative Solutions to Improve Herd Productivity
}

\author{
C. Arcidiacono, M. Barbari, S. Benni, E. Carfagna, G. Cascone, L. Conti, L. di \\ Stefano, M. Guarino*, L. Leso, D. Lovarelli, M. Mancino, S. Mattoccia, G. \\ Minozzi, S. M. C. Porto, G. Provolo, G. Rossi, A. Sandrucci, A. Tamburini, P. \\ Tassinari, N. Tomasello, D. Torreggiani and F. Valenti
}

M. Guarino $(*)$ - Department of Environmental Science and Policy, Università degli Studi di Milano, Via Celoria, 10-20133 Milan, Italy e-mail: marcella.guarino@unimi.it

D. Lovarelli - G. Provolo - A. Sandrucci - A. Tamburini - Department of Agricultural and Environmental Sciences, Production, Landscape, Agroenergy, Università degli Studi di Milano, Via Celoria, 2-20133 Milan, Italy

C. Arcidiacono - G. Cascone - M. Mancino - S. M. C. Porto - N. Tomasello · F. Valenti - Department of Agriculture, Food and Environment, Università degli Studi di Catania, Via S. Sofia, 100-95123 Catania, Italy

M. Barbari · L. Conti · L. Leso - G. Rossi - Department of Agriculture, Food, Environment and Forestry, Università degli Studi di Firenze, Via San Bonaventura, 1350145 Florence, Italy

S. Benni · P. Tassinari · D. Torreggiani - Department of Agricultural and Food Sciences, Università di Bologna, Viale Giuseppe Fanin, 50-40127 Bologna, Italy

G. Minozzi - Department of Veterinary Medicine, Università degli Studi di Milano, Via Celoria, 10-20133 Milan, Italy

E. Carfagna - Department of Statistical Sciences "Paolo Fortunati", Università di Bologna, Via Belle arti, 41, 40126 Bologna, Italy

L. di Stefano - S. Mattoccia - Department of Computer Science and Engineering, Università di Bologna, Viale Risorgimento 2, 40136 Bologna, Italy

\begin{abstract}
Among the most straining trends that farmers have to face there are: on one side, to guarantee welfare and adequate life conditions for animals and to reduce the environmental footprint, on the other side, to develop new strategies to improve farm management reducing costs. The current conditions and the expected developments of the dairy sector highlight a strong need for more efficient and sustainable farming systems. Studying heat stress, herd management and housing and animals' productive and reproductive performances is fundamental for the economic and environmental sustainability of the dairy chain.

New and effective tools to cope with these challenges have been provided by Precision Livestock Farming (PLF), which is nowadays increasingly applied and makes possible to control quali-quantitative parameters related to production, health, behaviour, and real-time locomotion per animal. The research key challenge is to turn these data into knowledge to provide real-time support in farming optimisation. This research focuses specifically on different systems to collect, process and derive useful information from data on animal welfare and productivity. A multi-disciplinary approach has been adopted to generate a decision support system for farmers.
\end{abstract}


Keywords Sustainability $\cdot$ Animal housing $\cdot$ Numerical modelling Efficient animal production $\cdot$ Precision livestock farming

\section{Introduction}

Heat stress, herd management and housing are of major concern for milk producers. These aspects enclose the main issues that farmers are expected to deal with in the near future under the economic, environmental and social points of view. The main driver to the attention to these topics is linked to the associated decrease in milk production and the subsequent large economic losses that all these aspects involve. In addition, the environmental and social sustainability of food products is widely increasing in importance.

Traditionally, research on heat stress, for example, has focused on tracking changes in physiology and health of the cows coping with the hot environment. However, to ensure high standards of welfare for dairy cattle, a broader approach is needed in order to evaluate the affective states and the housing and thermal managements (e.g., insulation, fans, misters and shower).

The development in technology has been giving new opportunities for monitoring cow's behaviour in real time, which is known as Precision Livestock Farming (PLF). PLF is very powerful because it applies principles of control engineering to optimising production and management processes of dairy cattle and uses the resulting models to monitor, predict and control single animals and herds allowing to introduce the most adequate solutions in every herd.

Studies that have been carried out on dairy cows in intensive breeding have shown that heat stress causes unusual behaviours, a decline in their fertility and a worsening of the quality and quantity of milk produced (Gernand et al. 2019; Porto et al. 2015). Therefore, heat stress is a key point for farmers, researchers and decision makers, and monitoring the response of animals to this aspect permits to identify the most adequate solutions to adapt to it.

Other aspects that can be monitored through PLF regard animal health and fertility (Arcidiacono et al. 2017a). During the oestrus phase, which precedes the fertility state, the motor activity undergoes a variable increase that ranges between $30 \%$ and $200 \%$ respect to the usual activity. The reduced profit margins in the sale of milk had focused farmers' attention towards the reproductive performance of the herd in order to minimise costs associated with the reproduction phase. Hence, identifying the fertile period of dairy cows is an increasingly pressing need for farmers (Arcidiacono et al. 2017b; Mayo et al. 2019). Though open source algorithms were successfully developed to automatically classify standing, walking, feeding and lying down behaviour of dairy cows, hardware design can be improved to be used in the severe environment conditions of dairy barns (Trnovszky et al. 2017). Therefore, building a prototype adoptable in PLF to early detect cows' oestrus is very important.

In regard of housing and animal welfare, recent studies showed that the lack of grazing is one of the major concerns of consumers respect to intensive livestock farming. Moreover, even if consumers revealed to be particularly sensible to this 
aspect, grazing has been almost completely abandoned in Italy. Therefore, potential benefits of allowing the cows to access pasture should be investigated. In particular, providing access to pasture can have the potential to improve various aspects of the sustainability in dairy farming, among which animal welfare and social acceptability of intensive farming (Bargo et al. 2002; Charlton and Rutter 2017). However, grazing may limit milk production in high yielding dairy cows, reduce the control over diet composition and result in exposure to unsuitable weather conditions. Since the housing conditions affect cattle welfare, the benefits of accessing to pasture and the willingness of the cows to access to outside areas (Legrand et al. 2009) may depend on the housing system.

Thegeneralgoalsofthisstudyaretoidentifytherelationshipsamongenvironmental conditions, fertility, behaviour and health state of the cow, and to find innovative solutions to ensure dairy cows' welfare and improve herd fertility by maintaining appropriateenvironmentalconditions. Toachievethis,comprehensivecow-

sidemeasurements are collected in order to obtain real-time responses to different managing solutions for housing and herd management and for high ambient temperatures that could be incorporated into heat abatement management decisions.

\section{Materials and Methods}

As shown in Fig. 1, the monitoring and processing of environmental parameters, behaviour and animal condition parameters represent the main points of the research.

Regarding the heat stress control, different solutions are analysed and compared to detect the animal behaviour. Eight herds that differ in design, materials, managing ventilation and cooling system are studied by measuring continuously several parameters and using wearable sensors and data loggers in the barn environment. These parameters include environmental conditions, animal activity and animal condition among which body temperature, heart rate, milk production and composition, milk somatic cell count, lameness, BCS and weight. Moreover, together with the analysis of other physiological parameters, individual monitoring of dairy-cow motor 

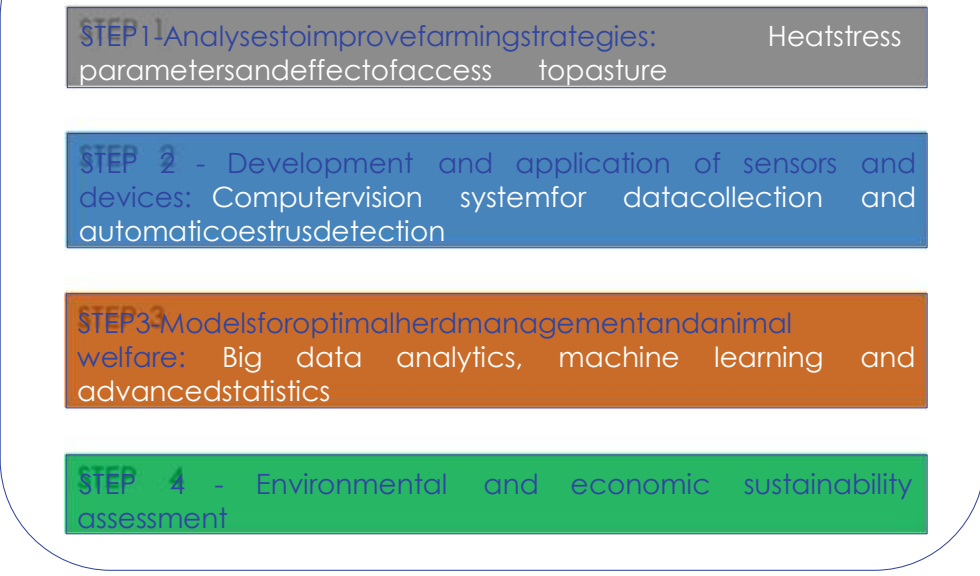

Fig. 1 Steps that define the phases of the project

activity may allow the early detection of some pathological and physiological status (Arcidiacono et al. 2017a), which also brings to a reduction in medical costs.

For the early detection of the oestrus phase, the commonly used inertial sensor based system is improved by adding hardware and software components for storing information regarding barn environmental conditions (air temperature, humidity ratio, air velocity, etc.) that are also modified by the management of different air cooling systems (evaporative or shower type with fans) usually adopted in hot climate regions (Arcidiacono et al. 2017b).

In regard of the animal welfare, a trial is carried out by providing animals the access to pasture in order to evaluate whether there is an improvement of welfare of dairy cows. This is performed comparing the cow response when present in two different typologies of dairy barns: the compost dairy barns (CDB) that showed to improve animal welfare and especially feet and leg health, and the conventional free stall barns (FSB). Cows in CDB are provided with an open bedded pack area for resting and walking, while in FSB there are individual stalls and concrete alleys. The effects of pasture access on the performance and welfare of lactating dairy cows housed in the two housing systems are monitored with PLF sensors, to monitor behaviour and health and to measure milk yield and quality. The experiments are repeated in different periods of the year and feeding cows with the same Total Mixed Ration (TMR) ad libitum inside the barn. The preference of cows to be indoor or on pasture is assessed by monitoring the number of cows inside and outside the barn through a multi-camera video recording system.

The collected data are used to develop a fuzzy logic model for assessing the risk of reduced productivity in relation to the animals' conditions and their environment. The economic cost and the environmental impact for every strategy for mitigating heat stress, evaluating the effect of pasture and improving herd management thank to sensors are compared using Life Cycle Analysis. Life Cycle Assessment and Life Cycle Costing will result with the environmental and economic impact of every investigated solution for understanding the most effective solution. 


\section{Expected Results and Applications}

The automatic systems and management techniques investigated during this project represent a series of innovative tools for the process of decision making that are in line with the recent trends of breeding widespread in Europe and favoured by the modern "smart farming". The monitoring and processing of big data is expected to furnish a large-scale modelling of animal behaviour, production and environmental conditions to keep into consideration specific livestock conditions and single animals of the herd. In particular, all collected data will be analysed as big data with advanced statistics and machine learning to develop models for real-time support.

From this research, it is expected also to identify and possibly adopt breeds of dairy cattle that are better equipped for hot environments, thus minimising the duration of compromised welfare. In addition, the comparison among data collected in the herds will show the differences related to the effect of heat stress, herd management and housing on milk production and animal health and welfare in the different contexts.

The scientific impact of the results will act in the field of PLF, since it will be possible to discern real-time data on the behavioural activities of dairy cows and facilitate the adoption of timely management protocols to safeguard animal health conditions and understand their physiological state. This will bring benefits and support to the decision making system. By promoting animal welfare, herd productivity will improve, as well as will increase the income of farmers and the environmental sustainability.

The effect on the economic and environmental points of view represents an essential aspect because consumers are increasingly demanding for sustainable intensive livestock farming systems and products. Additionally, also the social impact will emerge in regard of animal welfare because its achievement is a new requirement in those countries where animal husbandry technology has reached advanced levels that force animals to a very high productivity in environmental, physiological and food conditions that are, however, stressful.

Acknowledgements The activity presented in the paper is part of the research grant Progetti di Ricerca di rilevante Interesse Nazionale-Bando 2017 Prot. 20178AN8NC.

\section{References}

Arcidiacono, C., Porto, S. M. C., Mancino, M., \& Cascone, G. (2017a). Development of a threshold based classifier for real-time recognition of cow feeding and standing behavioural activities from accelerometer data. Computers and Electronics in Agriculture, 134, 124-134.

Arcidiacono, C., Porto, S. M. C., Mancino, M., \& Cascone, G. (2017b). A software tool for the automatic and real-time analysis of cow velocity data in free-stall barns: The case study of oestrus detection from Ultra-Wide-Band data. Biosystems Engineering, 173, 157-165.

Bargo, F., Muller, L. D., Delahoy, J. E., \& Cassidy, T. W. (2002). Performance of high producing dairy cows with three different feeding systems combining pasture and total mixed rations. Journal of Dairy Science, 85, 2948-2963.

Charlton, G. L., \& Rutter, S. M. (2017). The behaviour of housed dairy cattle with and without pasture access: A review. Applied Animal Behaviour Science, 192, 2-9.

Gernand, E., König, S., \& Kipp, C. (2019). Influence of on-farm measurements for heat stress indicators on dairy cow productivity, female fertility, and health. Journal of Dairy Science, 102, 6660-6671. 
Legrand, A. L., von Keyserlingk, M. A. G., \& Weary, D. M. (2009). Preference and usage of pasture versus free-stall housing by lactating dairy cattle. Journal of Dairy Science, 92, 36513658.

Mayo, L. M., Silvia, W. J., Ray, D. L., Jones, B. W., Stone, A. E., Tsai, I. C., et al. (2019). Automated estrous detection using multiple commercial precision dairy monitoring technologies in synchronized dairy cows. Journal of Dairy Science, 102, 2645-2656.

Porto, S. M. C., Arcidiacono, C., Anguzza, U., \& Cascone, G. (2015). The automatic detection of dairy cow feeding and standing behaviours in free-stall barns by a computer vision-based system. Biosystems Engineering, 133, 46-55.

Trnovszky, T., Kamencay, P., Orjesek, R., Benco, M., \& Sykora, P. (2017). Animal recognition system based on convolutional neural network. Digital Image Processing and Computer Graphics, 15(3), 517-525. 